\title{
GESTIÓN DEL CONOCIMIENTOY Y CAPACIDAD DE INNOVACIÓN EN UNA ORGANIZACIÓN RADIAL EN BARRANQUILLA
}

Knowledge Management and Innovation Capacity in a Radial Organization in Barranquilla

\author{
BETSY AQUINO VANEGAS \\ KARELIS BARRIOS HERNÁNDEZ \\ ENOHEMIT OLIVERO VEGA \\ JOSÉ MARÍA MENDOZA GUERRA
}

\section{RESUMEN}

Este capítulo aborda un análisis de la gestión del conocimiento y la capacidad de innovación en una organización radial de la ciudad de Barranquilla, con el fin de identificar cuáles son las condiciones facilitadoras para su desarrollo, al tiempo se caracteriza la capacidad de innovación y se reconoce el perfil de los innovadores de esta organización. La investigación reviste su mayor importancia porque se constituye en aporte significativo para avanzar en el tema de capacidad dinámica de innovación, del cual existen escasas evidencias. Desde el punto de vista teórico, se parte del enfoque de las capacidades dinámicas, para entender cómo la innovación es una fuente clave para poder adaptarse y responder a los cambios, y cómo contribuye a generar ventaja sostenible. Posteriormente, se establecen las condiciones de la gestión del conocimiento que facilitan su desarrollo. Se trata de una investigación descriptiva y exploratoria. La fuente primaria de recogida de datos, fue un cuestionario conformado por 17 ítems, medido en una escala Likert. Por último, se presentan los resultados y principales conclusiones de la investigación que dan evidencia del análisis del rol de la gestión del conocimiento, en el desarrollo de la capacidad de innovación.

Palabras clave: Gestión del conocimiento, capacidad de innovación, entorno, organización radial.

\section{ABSTRACT}

This chapter an analysis of knowledge management and innovation capacity in a radial organization in the city of Barranquilla, in order to identify the conditions that facilitate its development. The research is important because it constitutes a significant contribution to advance the concept of dynamic capacity for innovation, of which there is little evidence. From the theoretical point of view, it is based on 
the dynamic capabilities approach, to understand how innovation is a key source for adapting and responding to changes and how it contributes to generate sustainable advantage. Subsequently, the conditions of the knowledge management that facilitate its development are established. This is descriptive and exploratory research. The primary source of data collection was a questionnaire consisting of 17 items, measured in a likert scale, a descriptive analysis of the studied variables was carried out. Finally, we present the results and main conclusions of the research that give evidence of the role of knowledge management, in the development of innovation capacity.

Keywords: Knowledge management, innovation capacity, environment, radial organization.

\section{INTRODUCCIÓN}

En la actualidad se ha reconocido el rol que juega la gestión del conocimiento en la generación de valor y ventaja competitiva sostenible. Paralelamente, los cambios del entorno están obligando a trasformar la realidad empresarial, poniendo de relieve la importancia del conocimiento en la adaptación a las condiciones del entorno.

La forma como cada organización asume el dinamismo del entorno, depende de la rapidez de adaptación, la generación de ideas novedosas y el desarrollo de nuevas rutinas organizacionales que propicien procesos innovadores para responder a la turbulencia de este entorno (Barrios \& Olivero, 2015, 2017).

Siguiendo a Acosta \& Fischer (2013) en un mundo donde los mercados, productos, tecnología y competidores cambian de manera vertiginosa, la gestión del conocimiento en las empresas se convierte en un elemento clave de su éxito. Por tanto, los procesos mediante los cuales se crea y se gestiona el conocimiento constituyen la fuente de capacidades dinámicas y de ventaja competitiva sostenible (Camisón, 2002; Teece, Pisano, \& Shuen, 1997).

En este capítulo en particular se abordará la gestión del conocimiento y su relación con la capacidad de innovación en una organización radial de la ciudad de Barranquilla, partiendo del hecho que este tipo de organizacio- 
nes disponen de capacidades para convertir la información y el know-how en productos y servicios comercialmente viables (Barrios \& Olivero, 2015, 2017).

De acuerdo a Barrios \& Olivero (2015) las organizaciones construyen capacidad de innovación, cuando logran configurar y coordinar una serie de rutinas que propicien los procesos de transformación organizacional que requiere la innovación (Aguilar \& Yepes, 2006). Por tanto, la capacidad de innovación de una empresa es la habilidad que posee para cambiarse a sí misma, repetida y rápidamente (Shapiro, 2005), con el fin de seguir generando valor, transformando los procesos empresariales, para dar lugar a organizaciones más competitivas, ágiles y eficaces (Mathison et al., 2007; Barrios \& Olivero, 2015).

Se seleccionó como objeto de la investigación a una organización radial de la ciudad de Barranquilla; es una compañía 100 \% colombiana, que impulsa el desarrollo de las comunicaciones a través de la tecnología y la innovación. Además, hacen de la radio el escenario propicio para resaltar la diversidad cultural de las regiones, convirtiendo la comunicación publicitaria en plataforma para alcanzar el desarrollo sostenible de la industria y el comercio en nuestro país.

Esta organización nace gracias a la visión de sus fundadores que observaron en la radio un medio capaz de representar la expresión cultural y musical de quienes la escuchaban; generando la identidad necesaria para comunicar mensajes publicitarios exitosos, alcanzando rápida aceptación. Asimismo, la ampliación de su cobertura a las principales capitales del país, dieron paso al primer sistema de radio musical bailable del país. El liderazgo innovador de este proyecto impulsó el crecimiento del mercado de la radio musical de entretenimiento en Colombia.

Por otra parte, con la adaptación de los avances tecnológicos al servicio de las comunicaciones, la organización ha llevado sus marcas a internet, siendo pioneros en ofrecer a los oyentes el audio streaming de todas las emisoras, de tal manera que se valore la identidad de cada uno de los 
oyentes y se amplíe la cobertura de los mensajes de los anunciantes. Es así como el desarrollo creativo de los contenidos está ideado para llegar con mejor y más atractivos servicios a los oyentes y clientes.

Teniendo en cuenta la evolución anterior, la elección de esta organización como objeto de investigación, estuvo motivada por el interés de saber el rol que juega la gestión del conocimiento en el desarrollo de la capacidad de innovación y su relación con los resultados empresariales en este tipo de organización.

Con base en lo anterior, se pretende analizar el rol de la gestión del conocimiento en el desarrollo de la capacidad de innovación en una organización radial de la ciudad de Barranquilla, además de identificar cuáles condiciones favorecen su desarrollo.

\section{FUNDAMENTACIÓN TEÓRICA}

Antes de abordar el tema de capacidad de innovación, es necesario mencionar que el estudio sobre las capacidades dinámicas está en continua evolución, el fundamento de ellas se encuentra en el enfoque de recursos y capacidades. Los recursos han sido definidos por diferentes autores (Penrose, 1959; Schumpeter, 1978; Barney, 1991; Wernerfelt, 1984).

Penrose (1959) señala que el recurso más importante de una firma es la gerencia. Mientras Schumpeter (1978) considera que la función central de una empresa en el desenvolvimiento económico es hacer nuevas combinaciones de recursos. Por su parte, para Barney (1991) los recursos son heterogéneos e inmóviles entre las empresas, y propone que para que estos puedan tener el potencial de generar ventaja competitiva, deben ser: valiosos, raros, imperfectamente imitables e insustituibles.

Por otro lado, las capacidades se pueden concebir como la construcción de la sabiduría colectiva en cualquier organización (Garzón, 2013), y nacen a lo largo del tiempo mediante las interacciones que surgen entre los recursos de la empresa (Venture, 1994). Es así como una organización posee 
una capacidad cuando hace algo que es apreciado, y lo hace de manera efectiva, comparado con otras organizaciones menos capaces de obtener una solución (Dávila, 2013; Barrios \& Olivero, 2015).

Existen tres niveles de jerarquía de capacidades, en el primero se sitúan los recursos, en el segundo las capacidades y en el tercero y último las capacidades dinámicas, las cuales son las que dirigen a la organización a desarrollar ventaja competitiva sostenible en el largo plazo (Barrios \& Olivero, 2015; Aguilar \& Yepes, 2006; Winter, 2003; Garzón, 2013).

Barrios \& Olivero (2015) manifiestan que para entender cómo se crean ventajas competitivas sostenibles en entornos turbulentos, la literatura (Barney, 1991; Teece, Pisano, \& Shuen, 1997; Teece, 2007, 2009; Zott, 2003) ha demostrado que esto es posible a través del desarrollo de las capacidades dinámicas, las cuales permiten a la empresa reconfigurar su base de recursos y adaptarse a las condiciones cambiantes del mercado, con el objetivo de lograr una ventaja competitiva (Zahra \& George, 2002).

Es así como las capacidades dinámicas suponen un despliegue de capacidades insustituibles, intransferibles y difíciles de imitar como proceso estratégico de conformación, reconformación, configuración, reconfiguración y protección de la base de recursos y capacidades esenciales de una empresa, para la adaptación a las condiciones cambiantes del entorno y la generación de ventajas competitivas sostenibles (Barrios \& Olivero, 2015).

Por eso, en ocasiones la formación de capacidades dinámicas puede ser afectada por la cultura, la estructura y la estrategia desarrollada en el pasado, creando rigideces centrales (Aguilar \& Yepes, 2006; Leonard-Barton, 1992; Mendoza, 2013; Barrios \& Olivero, 2015).

En este sentido, las empresas que logran éxito en un ambiente competitivo son las que gestionan su conocimiento, para responder de manera rápida y repetitiva a las condiciones cambiantes del entorno. Las capacidades dinámicas son, por tanto, factores claves en la innovación y la optimización del curso estratégico global del futuro de la empresa. 
GESTIÓN DEL CONOCIMIENTO Y CAPACIDAD DE INNOVACIÓN

Modelos, Sistemas y Aplicaciones

\subsection{Gestión del conocimiento y capacidad dinámica de innovación}

En la era actual del conocimiento y la información, el factor estratégico de competitividad sostenible de las organizaciones es la gestión del conocimiento. Los conocimientos de las personas son la clave de la empresa y explican buena parte de la valoración que el mercado concede a una organización (Mejía \& Jaramillo, 2006).

En otras palabras, significa que lo más importante y estratégico para el desarrollo de una empresa es utilizar el talento humano como factor de generación de conocimiento productivo; finalmente este se constituye en la base de la innovación y la productividad (Nonaka \& Takeuchi, 1995) y, además, en fuente directa de la ventaja competitiva sostenible (Mejía \& Jaramillo, 2006).

En el contexto empresarial se puede afirmar que la innovación consiste en poner en el mercado un producto o servicio nuevo o mejorado acorde a la demanda de la sociedad (Castrillón, 1998). Esta, le permite a una organización crear valor a través del desarrollo de un nuevo conocimiento o por uso del conocimiento existente, mediante un proceso que comprende la definición de un problema o necesidad que debe ser atendida; la concepción de la idea que dará la solución, su adopción, su implementación y su comercialización (Becerra \& Álvarez, 2011).

Por tanto, la innovación implica un momento de destrucción creativa de conocimiento (Shumpeter, 1934) y de competencias existentes sobre todo cuando se trata de cambios radicales (Langlois \& Robertson, 2000). Esto no significa romper con toda la memoria organizacional, sino con algunos de sus aspectos. La disyuntiva que se presenta para la organización es explotar las rutinas existentes o bien, explorar nuevas rutinas (Mejía \& Jaramillo, 2006).

Es así como la innovación implica imaginación y creatividad de los individuos, empresas y sociedad en su conjunto, para predecir y enfrentar los retos del cambio (Mejía \& Jaramillo, 2006). Este proceso se materializa en nuevos productos o servicios, o por nuevos modelos de negocios, técni- 
cas administrativas y estructuras organizacionales (Jamrog, Vickers \& Bear, 2006; Becerra \& Álvarez, 2011) que respondan a los cambios impuestos por el entorno, para desarrollar la innovación como capacidad dinámica.

Para Wang \& Ahmed (2007) la capacidad dinámica de innovación es la habilidad de la empresa para el desarrollo de nuevos productos y servicios, métodos de producción, la identificación de mercados, el descubrimiento de fuentes de suministro y nuevas formas organizativas (Barrios \& Olivero, 2015).

Además, estos mismos autores expresan que un alto nivel de capacidad de innovación indica una respuesta a las cambiantes condiciones del mercado, siendo la empresa capaz de desarrollar nuevas ideas y transformarlas en productos, procesos o sistemas (Szeto, 2000). Es así como la capacidad de innovación es la aplicación del conocimiento relevante para obtener valor en el mercado y la implementación exitosa de ideas creativas dentro de una organización (Zhao, 2005).

Por tanto, la capacidad de innovación no solo requiere la explotación de conocimientos que impliquen la búsqueda de la novedad y la asunción de riesgo, o la exploración de conocimientos que pueden llegar a ser conocidos (March, 1991; Levinthal \& March, 1993). También supone mantener un desarrollo simultáneo entre los procesos de exploración y explotación, lo cual es explicado a través de la ambidestreza organizacional (Duncan, 1976) que deberá poseer la empresa para facilitar la innovación (Acosta \& Fischer, 2013).

La innovación como capacidad dinámica debe permitir un equilibrio entre las actividades de exploración y explotación de recursos y capacidades (Vivas, 2013; Acosta \& Fischer, 2013). En este sentido, estos surgen como proceso de intercambio entre los estímulos del entorno, los conocimientos que existen en la organización y las acciones de sus integrantes (Acosta \& Fischer, 2013).

De esta manera, el reto consiste en analizar qué rol juega la gestión del conocimiento en el desarrollo de esta capacidad, teniendo en cuenta que 
un factor de éxito de las organizaciones, en el contexto global que se vive actualmente en el mundo, es su capacidad para cambiar de manera apropiada, modificando no solo estructuras, sino también su portafolio de productos y servicios, modelo de negocio y su forma de gestión manteniendo una actitud de renovación y aprendizaje permanente (Barret, 1997; Font, et al., 1999; Berckhard, 1988; Calderón, Cuartas, \& Álvarez, 2009).

Para que las transformaciones trasciendan la idea de cambio y den paso a la innovación se requiere que actúen sobre supuestos enraizados en los colectivos humanos, para modificar actitudes y comportamientos de las personas (Calderón, Cuartas \& Álvarez, 2009). Es así como, la gestión del conocimiento juega un papel preponderante en el desarrollo de la capacidad dinámica de innovación. De acuerdo a la literatura (Bravo, 2005; Bravo \& Herrera, 2009) esta requiere de la gestión del conocimiento, a través de su creación (Nonaka \& Takeuchi, 1995), la absorción (Cohen \& Levinthal, 1990; Zahra \& George, 2002), la integración (Grant, 1996) y reconfiguración (Galunic, 1998; Lavie, 2006).

La creación y la absorción del conocimiento, indica que este se gestiona en la medida que los individuos que conforman la organización permanezcan en contacto con su entorno, de tal manera, que la percepción organizacional se oriente hacia el medio (Toffler, 1985; Barrios \& Olivero, 2015). La vigilancia del entorno supone que la organización por un lado, analiza y busca señales sobre innovaciones potenciales, amenazas y oportunidades de cambio e identifica aquellos factores o variables que constituyen oportunidades para la empresa, con el propósito de tomar ventaja de ellas y aprovecharlas para su desarrollo y, por otro lado, contrarrestar las posibles amenazas que enfrenta o deberá enfrentar, con el fin de neutralizar y minimizar su impacto negativo. Estas oportunidades y amenazas pueden presentarse en el contexto jurídico o normativo, económico, social, político, ambiental y cultural (Banguero, 2008; Barrios \& Olivero, 2015).

Es así como las personas pueden reconocer las tendencias, potencialidades y rupturas que afectarán el desarrollo futuro de la empresa, por lo 
que se requiere de conocimientos y las habilidades de estas para captar o desarrollar nuevas ideas y explorar el medio y a su vez establecer contacto efectivo y relaciones de colaboración con los actores del entorno (Mendoza, 2013; Barrios \& Olivero, 2015).

Sobre la absorción de conocimiento, varios estudios sobre la innovación consideran la capacidad de absorción como un elemento influyente en la capacidad para innovar (Knudsen \& Roman, 2004), debido a que se constituye como la habilidad y motivación de los empleados para obtener conocimiento externo y utilizarlo para el desarrollo de la capacidad de innovación. El concepto de capacidad de absorción fue introducido por Cohen \& Levinthal (1990) quienes mencionan que la absorción hace referencia a la habilidad para reconocer el valor de nueva información, asimilarla y aplicarla a fines comerciales.

Sin embargo, para gestionar el conocimiento, es necesario integrar el conocimiento de los individuos y de la organización, es decir se requiere de una previa interacción y colaboración entre las personas y el medio (Hung, Kao, \& Chu, 2008). La interacción enfatiza la utilización e intercambio de información entre unidades funcionales. La colaboración se fundamenta en el trabajo colectivo entre departamentos o entre organizaciones facilitando la capacidad de relacionamiento (Grant, 1996; Mendoza, 2013; Barrios \& Olivero, 2015). Por eso las capacidades internas existentes y su interacción con fuentes de conocimiento externo pueden afectar positivamente el nivel de innovación de las compañías (Cohen \& Levinthal, 1990; Bravo \& Herrera, 2009).

En consecuencia, la captación y desarrollo de ideas novedosas implican que la gestión del conocimiento fomenta la transformación y el cambio, a través del ba y la creatividad empresarial.

\section{2. $B a$}

El $\mathrm{Ba}$ ha sido definido como un contexto en el cual el conocimiento es compartido, creado y utilizado (Acosta, Zárate \& Fischer, 2014; Nishida, 1921; Shimizu, 1995). En este orden de ideas, el $B a$ se refiere a un espacio 
de conocimiento físico, virtual y mental o una combinación de todos estos para la generación de ideas nuevas y originales (Nonaka \& Konno, 1998).

Por tanto, el $\mathrm{Ba}$ constituye una ayuda para la solución de problemas dentro de una organización o un equipo de trabajo (Bueno, Rodríguez \& Salmador, 2008; Kanter, 1989; Acosta, 2010). De esta manera, las organizaciones desarrollan y dinamizan procesos encaminados a la creación de conocimiento a través de las interrelaciones de los individuos y grupos que generan innovaciones (Nonaka et al., 2000). En consecuencia, el Ba favorece el talento creativo de los individuos (Eskildsen et al., 1999; Acosta, 2010), creando un espacio de interacción entre la persona, el trabajo y el contexto de la empresa (Acosta, 2010).

Asimismo, a mayor número de ideas creativas generadas, mayor será el número de opciones disponibles para su implantación a la hora de innovar, y por ende, existirán más posibilidades de generación de nuevo conocimiento y de combinaciones del existente, y mayor será la flexibilidad de la organización para responder a las demandas y oportunidades externas. Por eso, gestionar el conocimiento implica la alineación del talento de las personas con los objetivos organizacionales, para promover la innovación.

\subsection{Creatividad Empresarial}

La creatividad es una condición indisociable de las personas. Por eso, la dirección debe procurar atraer trabajadores creativos animando su grado de participación y esfuerzo. Para ello es necesario originar un contexto de trabajo que refuerce la capacidad de aplicar la creatividad de los individuos a los problemas y situaciones relevantes de la organización (Cumming \& Oldham, 1997).

Pero las ideas, sugerencias y pensamientos de los empleados pueden quedar en sus cabezas sin dar lugar a posibles intercambios y conexiones, si no encuentran el ambiente propicio que los anime a explicitar sus aportaciones. Más aún, no basta con que los empleados aporten ideas rompedoras; será necesario su reconocimiento y difusión para maximizar su impacto en la innovación (Subramaniam \& Youndt, 2005). 
Por eso las ideas creativas constituyen en gran medida la materia prima a partir de las cuales se consiguen las innovaciones, y las empresas que presentan simultáneamente mucha creatividad y elevada capacidad de gestión de las innovaciones son las que tienen más probabilidades de éxito (Escorsa \& Valls, 2005).

La creatividad es entonces la acción de generar ideas nuevas y comunicarlas (De la Torre, 1997), por lo que se concibe como la capacidad de crear, producir, formar, dar nacimiento, dar origen o representar algo por primera vez (Gámez, 1998). Robbins y Judge (2009) manifiestan que la creatividad es una capacidad que permite la combinación de ideas nuevas y útiles, distintas a lo que se ha hecho antes, pero que sirven para resolver los problemas que se presentan (Barroso, 2012). Mientras, Clegg y Birch (2001) la consideran un factor de supervivencia ya que permite a las organizaciones adaptarse a su medio. Por su parte, De Bono (1996) afirma que la creatividad desempeña un papel importante en la construcción de alternativas de solución, pues si no hay creatividad, entonces solo es posible usar conceptos estandarizados que pueden no ser la mejor alternativa para las organizaciones (Barroso, 2012).

En este sentido la gestión del conocimiento, juega un papel fundamental en el desarrollo de la creatividad empresarial, teniendo en cuenta que se requiere de un clima laboral favorable, que fomente el cambio y la confianza. Además, aunque hoy día se reconoce la necesidad de contar con gente creativa, no se le prepara adecuadamente para ello (Barroso, 2012). Por tanto, falta estimular el pensamiento intuitivo mediante sesiones de resolución de problemas en las que se propicie la libre asociación y el uso de analogías, como indican Robbins y Judge (2009) y De Bono (1996), pero también el pensamiento crítico, ya que los buenos pensadores críticos suelen estar mejor preparados y equipados para la toma de decisiones y la resolución de problemas.

Por último, gestionar el conocimiento indica que se le debe reconfigurar constantemente. Schumpeter (1934) observó que algunas innovaciones 
consisten en extender las recombinaciones de recursos. En este sentido la reconfiguración de conocimiento se define como el proceso de generación de nuevas alternativas de configuración de recursos y capacidades con el fin de crear valor (Bravo \& Herrera, 2009).

De tal manera, del resultado de una gestión eficiente del conocimiento, se preparan respuestas creativas frente a los cambios del medio, esta respuesta se asocia también con la capacidad dinámica de innovación, porque se puede hablar de una respuesta incremental o radical (Mendoza, 2013).

\section{METODOLOGÍA}

La investigación desarrollada fue de tipo descriptiva y exploratoria puesto que permitió aplicar un conjunto de métodos y procedimientos científicos para recolectar datos puros, y generar estructuras de datos que describen el rol de la gestión del conocimiento en el desarrollo de la capacidad de innovación.

Este tipo de investigación es útil para identificar características del universo, señalando formas de conducta, establece comportamientos concretos y descubre y comprueba asociaciones entre las variables (Méndez, 2013).

Además, el carácter exploratorio se debe a que existen pocas evidencias de estudios al respecto y este trabajo servirá para la formulación de estudios con mayor nivel de profundidad.

La investigación se desarrolló en una organización radial, ubicada en la ciudad de Barranquilla, la cual es una compañía 100 \% colombiana que impulsa el desarrollo de las comunicaciones a través de la tecnología y la innovación. Hacen de la radio el escenario propicio para resaltar la diversidad cultural de las regiones, convirtiendo la comunicación publicitaria en plataforma para alcanzar el desarrollo sostenible de la industria y el comercio en nuestro país.

Para el caso de esta investigación la población está conformada por los 
empleados directos de la organización radial, los cuales son 77 empleados que ocupan cargos directivos, administrativos y operativos en la sede Barranquilla. La muestra se constituye por el 100 \% de la población. Ver Tabla 1, Ficha técnica de investigación.

Tabla 1. Ficha técnica de la investigación

\begin{tabular}{|c|c|}
\hline UNIVERSO DE LA INVESTIGACIÓN & ORGANIZACIÓN RADIAL \\
\hline Ámbito geográfico & Barranquilla, departamento del Atlántico \\
\hline Población & $\begin{array}{c}77 \text { empleados entre directivos, administrativos y } \\
\text { operativos }\end{array}$ \\
\hline Muestra & $100 \%$ de la población \\
\hline Técnica de recolección de la información & Encuesta \\
\hline Procesamiento de muestreo & Cuestionario enviado a empleados. \\
\hline Número de cuestionarios & 77 \\
\hline Tasa de respuesta & $100 \%$ \\
\hline Período de trabajo & Enero de 2015 - Septiembre de 2015 \\
\hline
\end{tabular}

Fuente: Elaboración propia

Para el logro de los objetivos propuestos se hizo necesaria la recolección de datos que permitieron obtener información útil y exacta, mediante la utilización de técnicas e instrumentos apropiados y confiables, los cuales fueron aplicados a los empleados de la organización radial.

La técnica utilizada para la recolección de datos de esta investigación es la encuesta y con ella se pretendió recoger información para analizar el rol que juega la gestión del conocimiento en el desarrollo de la capacidad de innovación en una organización radial de Barranquilla.

El instrumento elegido para la recopilación de la información ha sido un cuestionario. El cuestionario se compone de 17 ítems. Para ello, han sido determinantes aspectos como la claridad y la brevedad. Asimismo, con el 
fin de evitar el sesgo en las respuestas, estos ítems fueron organizados en un orden específico, de tal manera que los empleados respondieran en secuencia a las afirmaciones.

Previo a la presentación del cuestionario final, se realizó la operacionalización de las variables en el cuadro metodológico, donde se establecieron las variables a medir por la investigación con sus respectivos indicadores.

En lo referente a la medición del concepto capacidad de innovación y gestión del conocimiento, la redacción de las variables se ha basado en los resultados obtenidos de la revisión del marco teórico. De esta forma, se ha pretendido analizar el rol de la gestión conocimiento en el desarrollo de la capacidad de innovación.

Se utilizó una escala tipo Likert en el cuestionario (Siempre, casi siempre, algunas veces, casi nunca y nunca). De acuerdo a Padua (1979), este tipo de escala corresponde con un nivel de medición ordinal, consistente en una serie de ítems o juicios ante los cuales se solicita la reacción del sujeto. El estímulo (ítem o sentencia) que se presenta al sujeto, representa la propiedad que el investigador está interesado en medir (Garzon, 2013).

Por otra parte, a fines de lograr la validez y confiabilidad del instrumento de investigación, se utilizó el método de expertos y un estudio piloto. En el diseño del instrumento se cumplieron varias fases: en primer lugar, se elaboró un banco de ítems o preguntas, con posibilidades a ser utilizados en el instrumento final a aplicar. En segundo lugar se realizaron los filtros pertinentes por parte del tutor asignado, para finalmente obtener una propuesta establecida del posible instrumento a aplicar a la población objeto de estudio.

En tercer lugar, se realizó el análisis de validez por expertos, el cual incluyó la evaluación de la pertinencia o no pertinencia de los objetivos específicos y su relación con: las variables, las dimensiones, las subdimensiones, los indicadores y los ítems. Asimismo, evaluaron la redacción del instrumento elaborado. Según las sugerencias, hallazgos y recomendaciones expuestas por el comité de expertos, se realizaron los cambios perti- 
nentes en el instrumento de investigación, obteniendo como resultado el instrumento final aplicado a los empleados de la organización radial.

Finalmente, con el fin de confirmar la confiabilidad y validez del instrumento se aplicó un estudio piloto al 20 \% del total de la población, es decir a 15 empleados, el cual tuvo como propósito, además de determinar el nivel de confiabilidad del instrumento de investigación, validar la claridad, la lógica y la apariencia del mismo.

Por último, la metodología de análisis de los resultados, se realizó en dos secuencias: un análisis preliminar y un análisis descriptivo.

\section{RESULTADOS}

En este primer paso del análisis, se realizó la respectiva validación y edición, es decir, se verificó que los cuestionarios se hubieran rellenado de forma apropiada y completa. Asimismo, se revisó la codificación de las variables.

En el segundo paso se grabaron los datos en el paquete estadístico SPSS. Finalmente, se hizo la verificación final de errores con el paquete SPSS antes de proceder al análisis estadístico de los resultados.

\subsection{Análisis Descriptivo}

El sotfware estadístico SPSS, en el cual se grabaron los datos y se precodificaron, permitió la obtención de las tablas de ordenamiento y distribución de frecuencias. Inicialmente se realizó el análisis de fiabilidad el alfa de Cronbach, que permite averiguar la consistencia de una medida, es decir, la parte de dicha medida que está libre de error aleatorio, cuyo valor mínimo aceptable es 0,70 para poder afirmar que un factor multi-ítems es fiable (Cronbach, 1951). Para el caso de esta investigación el alfa de Cronbach arrojó un valor de 0,961, demostrando que la escala es altamente fiable. La investigación arrojó los siguientes resultados:

En lo que respecta a la capacidad de innovación, los resultados se relacionan con las evidencias teóricas hasta la fecha, esta se caracteriza por los 
procesos de exploración y ambidestreza organizacional, es decir, que una organización es capaz de explotar y explorar al mismo tiempo. En la Tabla 2 se puede observar que los empleados de la organización radial manifiestan en su mayoría que esta empresa siempre está explorando las condiciones cambiantes del entorno y es capaz de descubrir nuevas oportunidades de negocio a partir de sus relaciones con el sector externo. Paralelamente, esta organización puede desarrollar innovaciones teniendo en cuenta la base actual de sus recursos y capacidades.

Por otra parte, en lo que respecta a la gestión del conocimiento, la investigación demostró que en la organización radial, hay dos condiciones que propician su desarrollo, estas son: la creatividad empresarial y el $B a$.

La Literatura sobre creatividad empresarial, hace énfasis en que las ideas, sugerencias y pensamientos de los empleados pueden quedar en sus cabezas sin dar lugar a posibles intercambios y conexiones, si no encuentran el ambiente propicio que los anime a explicitar sus aportaciones. Más aún, no basta con que los empleados aporten ideas rompedoras, será necesario su reconocimiento y difusión para maximizar su impacto en la innovación (Subramaniam \& Youndt, 2005). En consecuencia, en la Tabla 3 se evidencia que en la organización radial se fomenta la generación de ideas novedosas y su socialización. Asimismo, la dirección de talento humano influye de manera positiva mediante la existencia de un programa formalizado que recoge los objetivos, competencias, responsabilidades y desarrollo del puesto. Al tiempo, la empresa está desarrollando aprendizaje a partir de la experiencia de sus empleados, proveedores y clientes para desarrollar innovaciones. No obstante, no existen incentivos ligados a la innovación, lo que en gran medida limita el proceso. 


\begin{tabular}{|c|c|c|c|c|c|c|c|}
\hline $\begin{array}{l}\mathbb{J} \\
\sum \\
z\end{array}$ & $\begin{array}{l}\circ 0 \\
\text { Oे }\end{array}$ & $\begin{array}{l}\circ \\
\text { Oे } \\
\text { Oे }\end{array}$ & $\begin{array}{l}\circ 0 \\
\text { Oे }\end{array}$ & $\begin{array}{l}\stackrel{0}{0} \\
\text { Oे }\end{array}$ & 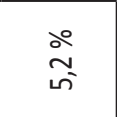 & $\begin{array}{l}\stackrel{0}{0} \\
\text { Oे }\end{array}$ & $\begin{array}{l}\stackrel{0}{0} \\
\text { Oे }\end{array}$ \\
\hline 至尤 & $\begin{array}{l}\stackrel{\circ}{0} \\
\stackrel{i}{\sim}\end{array}$ & 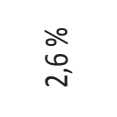 & $\begin{array}{l}\stackrel{0}{0} \\
\grave{i}\end{array}$ & $\begin{array}{l}\stackrel{\circ}{~} \\
\text { مे }\end{array}$ & 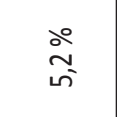 & $\begin{array}{l}\text { ळे } \\
\text { مे }\end{array}$ & $\begin{array}{l}\circ 0 \\
\text { Oे }\end{array}$ \\
\hline 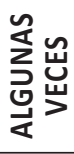 & $\stackrel{\circ}{\stackrel{m}{\approx}}$ & $\begin{array}{l}\text { ㅇ } \\
\stackrel{\tilde{n}}{\tilde{m}}\end{array}$ & $\begin{array}{l}\text { ১0 } \\
\text { bे } \\
\text { Dે }\end{array}$ & $\begin{array}{l}\stackrel{\circ}{\sim} \\
\stackrel{d}{\sim}\end{array}$ & \begin{tabular}{l} 
do \\
\multirow{0}{0}{} \\
Oे
\end{tabular} & $\frac{\stackrel{a}{a}}{\stackrel{a}{a}}$ & $\begin{array}{l}\text { مे } \\
\text { مे }\end{array}$ \\
\hline 首容 & $\begin{array}{l}\text { ১0 } \\
\text { m. } \\
\text { mె }\end{array}$ & $\begin{array}{l}\stackrel{\circ}{\stackrel{2}{*}} \\
\stackrel{f}{*}\end{array}$ & $\begin{array}{l}\stackrel{0}{0} \\
\stackrel{\sigma}{\sigma} \\
\stackrel{y}{*}\end{array}$ & $\begin{array}{l}\stackrel{0}{2} \\
\stackrel{f}{f}\end{array}$ & $\frac{\stackrel{\circ}{N}}{\hat{m}}$ & $\begin{array}{l}\stackrel{\circ}{\circ} \\
\text { مे }\end{array}$ & 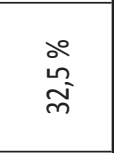 \\
\hline$\frac{w}{\stackrel{u}{a}}$ & \begin{tabular}{l} 
do \\
\multirow{d}{0}{} \\
di
\end{tabular} & $\begin{array}{l}\text { o } \\
\infty \\
\text { Dิ }\end{array}$ & $\begin{array}{l}\stackrel{\circ}{m} \\
\stackrel{\sim}{\sim}\end{array}$ & $\stackrel{\circ}{\stackrel{m}{\sim}}$ & 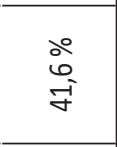 & $\frac{\stackrel{0}{-1}}{\stackrel{\sim}{\sim}}$ & 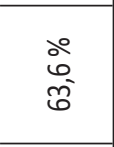 \\
\hline 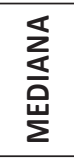 & $\underset{f}{\stackrel{f}{\circ}}$ & $\underset{+}{\stackrel{8}{+}}$ & $\begin{array}{l}8 \\
\stackrel{+}{*}\end{array}$ & $\begin{array}{l}8 \\
\stackrel{\forall}{*}\end{array}$ & $\begin{array}{l}8 \\
\stackrel{+}{*}\end{array}$ & $\underset{\leftarrow}{\stackrel{8}{\circ}}$ & 음 \\
\hline 号 & $\curvearrowright$ & 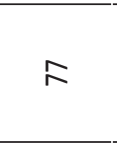 & 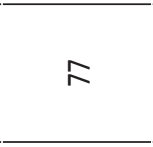 & $\approx$ & 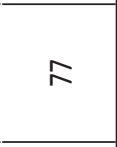 & 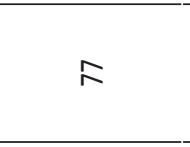 & $\curvearrowright$ \\
\hline$\dot{0}$ & $\approx$ & 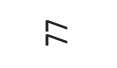 & 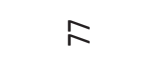 & 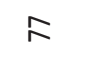 & $\approx$ & 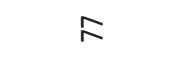 & $\approx$ \\
\hline 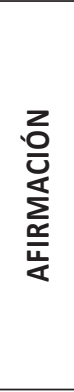 & 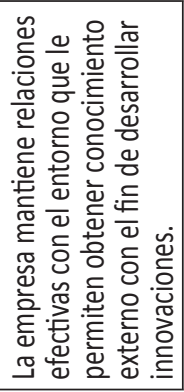 & 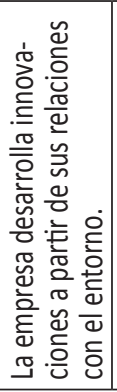 & 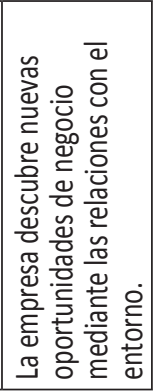 & 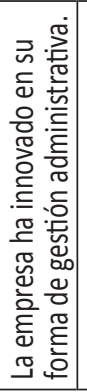 & 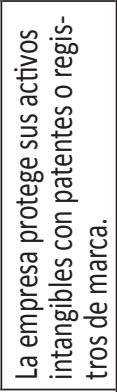 & 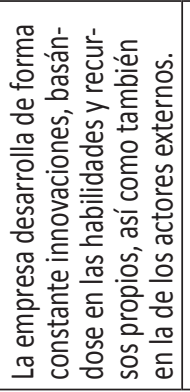 & 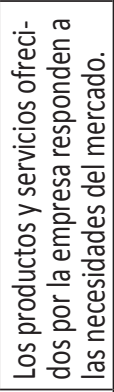 \\
\hline 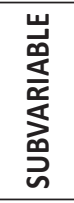 & \multicolumn{5}{|c|}{$\begin{array}{l}\text { :음 } \\
\frac{\pi}{0} \\
\frac{0}{2} \\
\text { 준 }\end{array}$} & \multicolumn{2}{|l|}{ 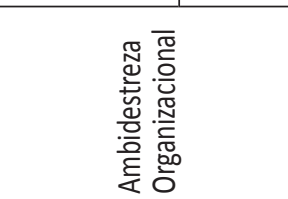 } \\
\hline 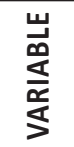 & \multicolumn{7}{|c|}{ 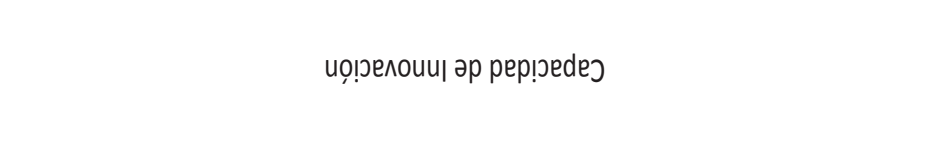 } \\
\hline
\end{tabular}


GESTIÓN DEL CONOCIMIENTO Y CAPACIDAD DE INNOVACIÓN

Modelos, Sistemas y Aplicaciones

\begin{tabular}{|c|c|c|c|c|c|c|}
\hline 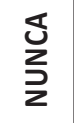 & ○̊ & ஃ̊ & $\stackrel{\circ}{\rightarrow}$ & ๙̊ & 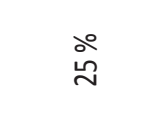 & $\stackrel{\circ 0}{ }$ \\
\hline 产起 & $\stackrel{\text { ○े }}{\text { mे }}$ & $\begin{array}{l}\stackrel{0}{\infty} \\
\infty\end{array}$ & $\begin{array}{l}\stackrel{\circ}{~} \\
\text { تे }\end{array}$ & $\stackrel{\text { ㅇ }}{\stackrel{7}{ }}$ & $\begin{array}{l}\text { ㅇ } \\
\text { ㄱ }\end{array}$ & $\begin{array}{l}\text { ㅇ } \\
\text { ㄱ }\end{array}$ \\
\hline 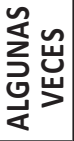 & $\begin{array}{l}\text { Әे } \\
\text { Әे }\end{array}$ & $\stackrel{\stackrel{\circ}{\curvearrowright}}{\stackrel{\Delta}{N}}$ & $\stackrel{\circ}{\vec{N}}$ & $\stackrel{\circ}{\vec{\sim}}$ & $\begin{array}{l}\text { ㅇ } \\
\text { నे }\end{array}$ & 吕 \\
\hline 㐫岕 & $\begin{array}{l}\text { ১े } \\
\text { వे }\end{array}$ & ஓे & $\stackrel{\stackrel{\circ}{\sharp}}{\stackrel{\gamma}{2}}$ & $\begin{array}{l}\stackrel{\circ}{\forall} \\
\vec{\forall}\end{array}$ & $\stackrel{\circ}{\infty}$ & 웅 \\
\hline 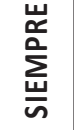 & ○े & $\begin{array}{l}\stackrel{0}{~} \\
\text { ને }\end{array}$ & $\begin{array}{l}\stackrel{\otimes}{\sim} \\
\stackrel{\sim}{2}\end{array}$ & $\begin{array}{l}\text { 움 } \\
\text { ஸे }\end{array}$ & 웅 & ㅇํํ \\
\hline 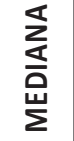 & $\begin{array}{l}8 \\
\stackrel{+}{+}\end{array}$ & $\underset{\forall}{\stackrel{8}{+}}$ & $\underset{\forall}{\stackrel{8}{+}}$ & $\underset{+}{\stackrel{8}{+}}$ & $\underset{\sim}{\text { O }}$ & $\underset{m}{\stackrel{8}{0}}$ \\
\hline $\begin{array}{l}\text { 号 } \\
\text { 荌 }\end{array}$ & 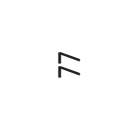 & 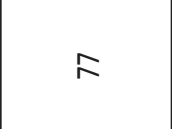 & 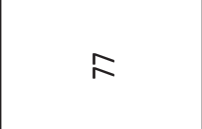 & 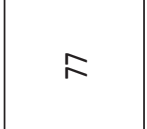 & $\AA$ & 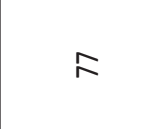 \\
\hline i & $\approx$ & 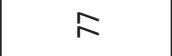 & $\approx$ & $\approx$ & $\curvearrowright$ & $\approx$ \\
\hline 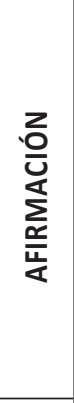 & 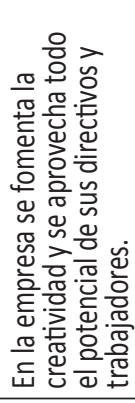 & 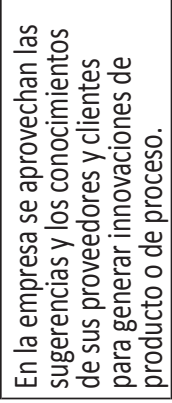 & 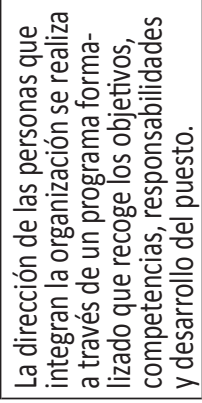 & 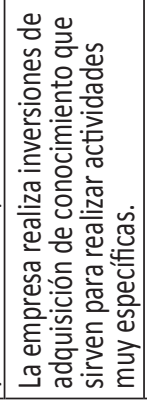 & 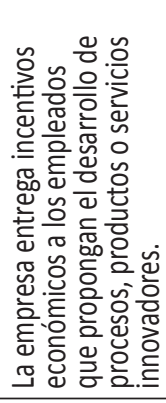 & 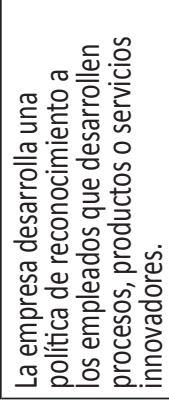 \\
\hline 的岂 & \multicolumn{6}{|c|}{ 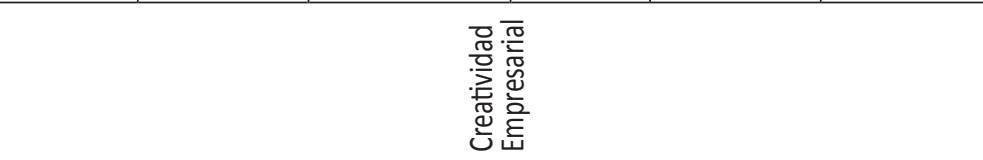 } \\
\hline 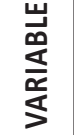 & \multicolumn{6}{|c|}{ 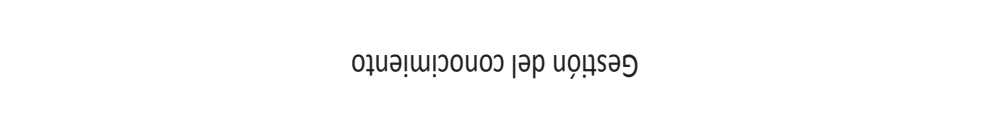 } \\
\hline
\end{tabular}


En la Tabla 4. Se puede demostrar que en la organización, en algunas ocasiones los empleados comparten su experiencia para generar innovaciones. Igualmente, se evidenció que si bien el nivel de formación de los integrantes facilita asumir nuevos cambios, estos no siempre tienen un alto nivel de imaginación. Por otra parte, los empleados consideran que sus sugerencias, las de los proveedores y clientes se tienen en cuenta para desarrollar nuevas prácticas, productos o servicios. Se resalta en los resultados que la organización invierte regularmente en tecnología de punta y los empleados consideran que disponen de medios y recursos suficientes para realizar su trabajo.

De esta manera, los resultados generales evidenciados muestran que las condiciones de la gestión del talento humano que propician el desarrollo de la capacidad de innovación, a través de los procesos simultáneos de exploración, explotación y por ende de la ambidestreza organizacional, son: la creatividad empresarial y el espacio compartido de conocimiento $B a$.

Por otra parte, la investigación evidenció que en la organización no existe una política formal para la entrega de incentivos económicos y no económicos ligados a la innovación y que además en el proceso de selección no se han formalizado las características que debe tener un nuevo empleado para que fomente la innovación en la empresa.

\section{CONSIDERACIONES FINALES}

El objetivo de esta investigación ha sido analizar el rol de la gestión conocimiento en el desarrollo de la capacidad de innovación en una organización radial de la ciudad de Barranquilla. Iniciamos realizando una revisión sobre las capacidades dinámicas, en particular la capacidad de innovación y las principales aportaciones de diferentes autores, para luego reconocer una definición para la investigación del constructo capacidades dinámicas. Posteriormente se hace un análisis detallado de las bases teóricas sobre la gestión del conocimiento y sus condiciones facilitadoras en el desarrollo de la innovación. 
GESTIÓN DEL CONOCIMIENTO Y CAPACIDAD DE INNOVACIÓN

Modelos, Sistemas y Aplicaciones

\begin{tabular}{|c|c|c|c|c|c|c|c|c|c|c|c|}
\hline $\begin{array}{l}\text { ঠ } \\
\text { ż } \\
\text { z }\end{array}$ & $\stackrel{\circ}{r}$ & ஃ̊ & ஃ̊ & $\stackrel{\circ}{\stackrel{\circ}{r}}$ & $\stackrel{\circ}{\circ}$ & in & in & ○̊ & $\stackrel{\circ}{\circ}$ & ஃ̊ & ஃ̊ \\
\hline দ্রু & $\stackrel{\stackrel{\circ}{m}}{\rightarrow}$ & $\stackrel{\circ}{r}$ & $\begin{array}{l}\text { வ } \\
\infty\end{array}$ & $\stackrel{\circ}{m}$ & $\stackrel{\circ}{\stackrel{\circ}{\rightarrow}}$ & $\stackrel{\text { ळo }}{\text { m }}$ & $\stackrel{\circ}{\stackrel{\circ}{f}}$ & $\begin{array}{l}\stackrel{\circ}{\Delta} \\
\stackrel{\forall}{+}\end{array}$ & $\begin{array}{l}\text { வ } \\
\infty\end{array}$ & $\stackrel{\circ}{\stackrel{\sim}{二}}$ & $\stackrel{\circ}{\stackrel{\circ}{f}}$ \\
\hline 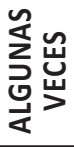 & $\begin{array}{l}\text { ১े } \\
\text { ర్ల }\end{array}$ & $\begin{array}{l}\stackrel{\circ}{\sim} \\
\stackrel{2}{\sim}\end{array}$ & $\begin{array}{l}\stackrel{\circ}{m} \\
\vec{m}\end{array}$ & $\stackrel{\circ}{\stackrel{\sigma}{\sigma}}$ & i̊ & $\stackrel{\circ}{\stackrel{2}{ }}$ & $\begin{array}{l}\text { वे } \\
\vec{m}\end{array}$ & @̊ & $\begin{array}{l}\text { ১0 } \\
\text { bे }\end{array}$ & $\begin{array}{l}\text { do } \\
\text { o }\end{array}$ & $\stackrel{\circ}{\approx}$ \\
\hline 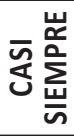 & $\begin{array}{l}\stackrel{\circ}{m} \\
\stackrel{m}{-1}\end{array}$ & $\begin{array}{l}\text { ஃ̊ } \\
\text { y }\end{array}$ & $\stackrel{\circ}{\underset{\Im}{\Im}}$ & $\stackrel{\text { ळे }}{\text { m }}$ & $\begin{array}{l}\text { ஃ̊ } \\
\text { ९े }\end{array}$ & $\begin{array}{l}\stackrel{\circ}{\infty} \\
\text { po }\end{array}$ & $\begin{array}{l}\stackrel{\circ}{ } \\
\stackrel{\nu}{n}\end{array}$ & ㅇํํ & 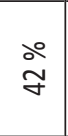 & 吕 & $\begin{array}{l}\text { ஃ̊ } \\
\text { 今े }\end{array}$ \\
\hline 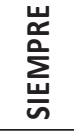 & 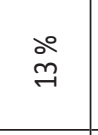 & 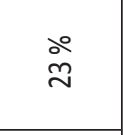 & 유 & 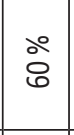 & $\begin{array}{l}\text { ळ̊ } \\
\text { ñ }\end{array}$ & $\stackrel{\circ}{\stackrel{一}{~}}$ & $\stackrel{\circ}{\approx}$ & $\stackrel{\circ}{\stackrel{\sim}{*}}$ & $\stackrel{\circ}{\stackrel{\circ}{7}}$ & $\stackrel{\circ}{\dddot{\eta}}$ & $\stackrel{\circ}{\approx}$ \\
\hline 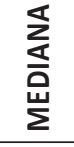 & $\underset{m}{8}$ & $\begin{array}{l}8 \\
+\end{array}$ & $\begin{array}{l}\text { \& } \\
+\end{array}$ & 焉 & 焉 & $\begin{array}{l}8 \\
\stackrel{+}{*}\end{array}$ & ষ্লি & $\underset{\sim}{\stackrel{8}{+}}$ & $\begin{array}{l}8 \\
\underset{f}{0}\end{array}$ & $\underset{m}{\stackrel{\text { }}{\text { - }}}$ & $\begin{array}{l}8 \\
\stackrel{+}{*}\end{array}$ \\
\hline 另 & 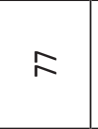 & 尺 & 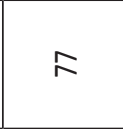 & 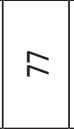 & 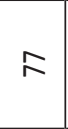 & 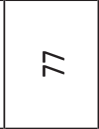 & 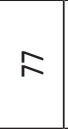 & 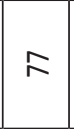 & 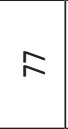 & 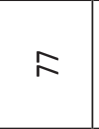 & 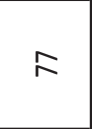 \\
\hline$\dot{0}$ & $\curvearrowright$ & 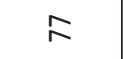 & $\curvearrowright$ & 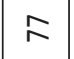 & $\curvearrowright$ & $\curvearrowright$ & 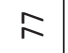 & $\approx$ & 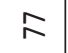 & 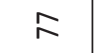 & $\curvearrowright$ \\
\hline 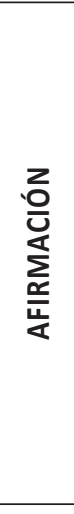 & 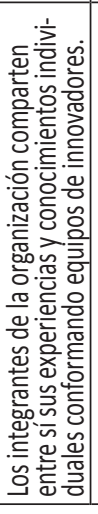 & 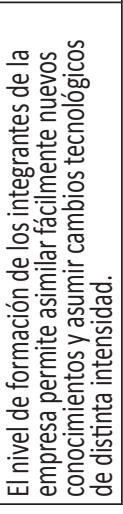 & 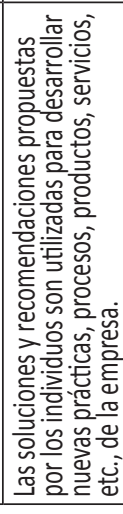 & 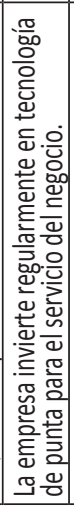 & 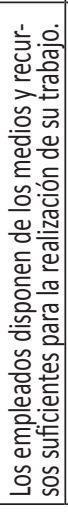 & 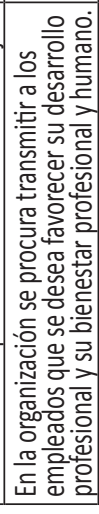 & 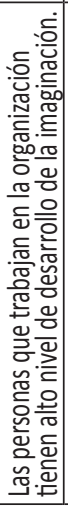 & 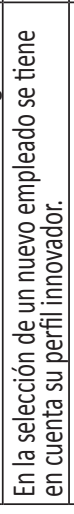 & 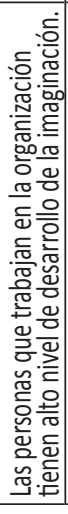 & 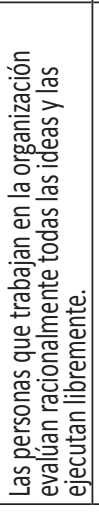 & 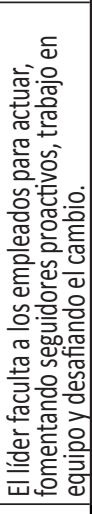 \\
\hline 岁 & \multicolumn{11}{|c|}{$\check{\infty}$} \\
\hline 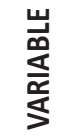 & \multicolumn{11}{|c|}{ 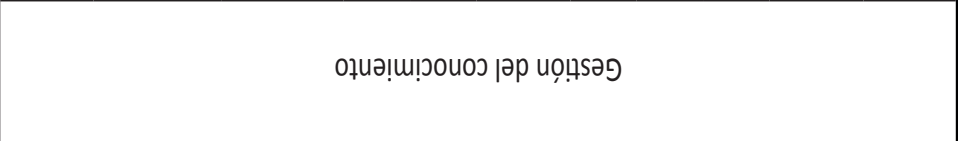 } \\
\hline
\end{tabular}


Una vez definida la capacidad dinámica de innovación y la gestión del conocimiento, se presenta el diseño de la investigación y la técnica de obtención de los datos, que permitió la medición de las variables, las cuales se plantearon en dos grupos a partir de la revisión exhaustiva de la literatura: por una parte, está la variable Capacidad de innovación y por otra, la variable Gestión del conocimiento.

El análisis estadístico se realizó en dos secuencias: análisis preliminar de los datos, un análisis descriptivo de los resultados, para lo cual se utilizó el SPSS.

La variable Capacidad de innovación se abordó desde el desarrollo de procesos simultáneos de exploración y explotación en la organización que dan lugar a la ambidestreza organizacional, sobre esto la empresa objeto de estudio demostró que es capaz de desarrollar ambos procesos al tiempo y que sus productos y servicios responden a las necesidades del entorno y que en gran medida se debe a la capacidad que tienen de reconocer oportunidades del medio y a sus relaciones efectivas con actores externos.

La variable Gestión del conocimiento se estudió desde las condiciones facilitadoras para el desarrollo de la innovación, encontrando que estas son: la creatividad empresarial y el espacio compartido de conocimiento $\mathrm{Ba}$.

No obstante, en esta variable se evidenció la carencia de las políticas formales de la organización para la entrega de incentivos económicos y no económicos al personal por el desarrollo de innovaciones.

Este análisis sobre la gestión del conocimiento en el desarrollo de la capacidad de innovación confirma que el rol de la gestión humana es preponderante en el desarrollo de la innovación como una capacidad dinámica en la organización radial para responder a los cambios vertiginosos del entorno. 
GESTIÓN DEL CONOCIMIENTO Y CAPACIDAD DE INNOVACIÓN

Modelos, Sistemas y Aplicaciones

\section{REFERENCIAS BIBLIOGRÁFICAS}

Acosta, J. (2010). Creación y desarrollo de capacidades tecnológicas: un modelo de análisis basado en el enfoque de conocimiento. (Tesis doctoral). Madrid, España: Universidad Autónoma de Madrid.

Acosta, J., \& Fischer, A. (2013). Condiciones de la gestión del conocimiento, capacidad de innovación y resultados empresariales. Un modelo explicativo. Pensamiento y Gestión, (35).

Acosta, J., Longo-Samoza, M., \& Fischer, A. (2013). Capacidades dinámicas y gestión del conocimiento en nuevas empresas de base tecnológica. Cuadernos de Administración, 26(47), 35-62.

Acosta, J., Zárate, R., \& Fischer, A. (2014). Ba: espacio de conocimiento. Contexto para el desarrollo de capacidad de innovacion. Un análisis desde la gestión del conocimiento. Revista de Escuela de Administración de Negocios, 76, 44-63.

Aguilar, J., \& Yepes, E. (2006). Gestión de capacidades dinámicas e innovación: Una aproximación conceptual. Revista de Ciencias de Administración, 8(16), 1-15.

Banguero, H. (2008). Un marco conceptual para el análisis del entorno económico de la empresa. Revista El hombre y la Máquina, (30), 64-77. Universidad Autónoma de Occidente

Barney, J. (1991). Firm resources and Sustained Competitive Advantage. Journal of Management, 17(1), 99-120.

Barrett, R. (1997, April). Liberating the corporate soul. HR Focus, 74(4), 15-16.

Barrios-Hernández, K., \& Olivero-Vega, E. (2015). La innovación en instituciones de educación superior: Un modelo basado en capacidades dinámicas. Barranquilla, Colombia: Universidad Simón Bolívar.

Barrios-Hernández, K., Olivero-Vega, E. \& Acosta-Prado, J. (2017). Capacidad dinámica de innovación en instituciones de educación superior. Revista Espacios, 38(01), 1-24.

Barroso, F. (2012). Factores y razones para desarrollar la creatividad en las 
empresas. Un estudio en el Sureste de México. Revista de Ciencias Sociales XVIII(3), 509-516.

Becerra, F., \& Álvarez, C. (2011). El Talento Humano y la Innovación Empresarial en el Contexto de las Redes Empresariales: El Clúster de Prendas de Vestir en Caldas- Colombia. Estudios Gerenciales, 119(27), 209-225.

Beckhard, R. (1988). The executive management of transformational change. In R. Kilmann, H. \& Covin, T. J. (Eds.), Corporate transformation: Revitalizing organizations for a competitive world (pp. 89-101). San Francisco: Jossey-Bass.

Bravo, E. (2005). Identificación y caracterización de las capacidades dinámicas que intervienen en el contexto de la innovación de producto (estudio de casos). Barcelona: Universidad Politécnica de Cataluña.

Bravo, E., \& Herrera, L. (2009). Generación de capacidades dinámicas mediante la innovación organizacional: Un múltiple estudio de casos exploratorio. 3rd International Conference on Industrial Engineering and Industrial Management, Barcelona.

Bueno, E., Rodríguez, J. \& Salmador, M.P. (2008). Knowledge creation as a dynamic capability: implications for innovation management and organisational design. International Journal Management Practice, 2(1), $72-82$

Calderón, G., Cuartas, G., \& Álvarez, C. (2009). Transformación organizacional y prácticas innovadoras de gestión humana. Innovar, 35(19), 151-166.

Camisón, C., \& Julián, B. (2008). Factores de la capacidad de absorción de conocimiento: Un estudio empírico. España: Universisitat de Jaumei.

Camisón, C. (2002). Las competencias distintivas basadas en activos intangibles. En P. Morcillo y J. Fernández (Eds). Nuevas claves para la dirección estratégica (pp.117-151). Barcelona: Ariel Economía.

Chesbrough, H. (2006). Innovación Abierta. Barcelona: Plataforma Editorial. Chesbrough, H. Vanhaverbeke, W. West, J. (2006). Open Innovation: Reaching a New Paradigm. Estados Unidos: Oxford University Press. 
Claver, E., Llopis, J., García, D. \& Molina, H. (1998). Organizational culture for innovation and new technological behavior. The Journal of High Technology Management Research, 9(1), 55-68.

Clegg, B. \& Birch, P. (2001). Creatividad al Instante. México: Granica.

Cohen, W., \& Levinthal, D. (1990). Absorptive Capacity: A new perspective on learning and innovation. Adminsitrative Science Quaterly, 35(1), 128-152.

Cronbach, L. J. (1951). Coefficient alpha and the internal structure of tests. Psychometrika, 16, 297-334.

Cummings, A., \& Oldham, G. R. (1997). Enhancing creativity: Managing work contexts for the high potential employee. California Management Review, 40, 22-38.

Dávila L. J. (2013). Capacidades organizacionales: dinámica por naturaleza. Cuadernos de Administración, 26(47), 11-33.

De Bono, E. (1996). El Pensamiento Creativo. México: Editorial Paidós Mexicana.

De la Torre, S. (1997). Creatividad y Formación: Identificación, Diseño y Evaluación. México: Trillas.

Duncan, R. B. (1976). The ambidextrous organization: Designing dual structures for innovation. In R. H. Kilmann, L. R. Pondy, and D. Slevin (Eds.), The management of organization design: Strategies and implementation. 167-188. New York: North Holland.

Escorsa, P., \& Valls, J. (2005). Tecnología e innovación en la empresa. México D.F: Alfa Omega Grupo Editor Sa de CV.

Eskildsen, J. K., Dahlgaard, J. J. y Norgaard, A. (1999). The impact of creativity and learning on business excellence. Total Quality Management, 10(4-5), 523-530.

Evan, W.M. \& Black, G. (1967). QQRYDWLRQ LQ EXVLQHVV RUJDQL]Dtions: Some factors associated with success or failure. The Journal of Business, 40(4), 519-530.

Font, I., Gudiño, P., Sánchez, A., Córdova, A. O. \& Morales, A. (1999, ene- 
ro-junio). El administrador frente a la necesidad de transformación organizacional. Gestión y estrategia, 15, UAM-A. Extraído el 9 de mayo desde: http://www.azc. uam.mx/publicaciones/gestion/num15/doc07.htm

Galunic, D. C. (1998). Resource recombinations in the firm: Knowledge structures and the potential for Schumpeterian innovation. Strategic Management Journal, 19(12), 1193-1201.

Garzón, M. (2013). Avance del proyecto de investigación. La sostenibilidad de las Pymes familiares de Barranquilla. Barranquilla, Colombia: Universidad EAN.

Garzón, M. (2013). El constructo capacidades dinámicas. Ide@s CONCYTEG, 8(99).

Grant, R. (1996). Prospering in dynamically-competitive environments: Organizational capability as knowledge integration. Organization Science, 7(4), 375-387.

Gámez, G. (1998). Todos Somos Creativos. Barcelona: Ediciones Urano.

Hotz-Hart, B. (2000). Innovation networks, regions and globalization. En G. Clark, M. Feldman y M. Gertler (Eds.), The Oxford Handbook of Economic Geography. 432-450. Oxford: Oxford University Press.

Hung, H. F., Kao, H. P., \& Chu, Y. Y. (2008). An empirical study on knowledge integration, technology innovation and experimental practice. Expert Systems with Applications, 35(1), 177-186.

Jamrog, J., Vickers, M. \& Bear, D. (2006). Building and sustaining a culture that supports innovation. Human Resources Planning, 29(3), 9-19.

Kanter (1989): "When a Thousand Flowers Bloom: structural, collective and social conditions for innovation in organizational design". En Myers, P.S. (eds.) (1996): Knowledge Management Organizational Design. Butterworth-Heinermann

Knudsen, H. K., \& Roman, P. M. (2004). Modeling the use of innovations in private treatment organizations: The role of absorptive capacity. Journal of Substance Abuse Treatment, 26(1), 353-361.

Langlois, R., \& Robertson, P. (2000). Empresas, mercados y cambio económico. Barcelona: Biblioteca Episteme. 
Lavie, D. (2006). Capability reconfiguration: An analysis of incumbent responses to technological change. Academy of Management Review, 31(1), 153-174.

Leonard-Barton, D. (1992). Core capabilities or core rigidities: a paradox in managing new product development. Strategic Management Journal, 13, 111-125.

Levinthal, D., \& March, J. (1993). The myopia of learning. Management Journal, 14, 95-112.

March, J. (1991). Exploration and exploitation in organizational learning. Organization Science, 2, 71-87.

Mathison, L., Gándara, J., Primera, C. \& García, L. (2007). Innovación: factor clave para lograr ventajas competitivas. Negotium, 3(7), 65-83.

Mejía, G., \& Jaramillo, M. (2006). Formación del talento humano: factor estratégico para el desarrollo de la productividad y la competitividad sostenibles en las organizaciones. Revista Científica Guillermo de Ockham, 1(4), 43-81.

Méndez, C. (2013). Metodología: Diseño y desarrollo del proceso de investigación con énfasis en ciencias empresariales. México D.F.: LIMUSA.

Mendoza, J. M. (2013). La Capacidad dinámica de ripostar en la empresa: Confrontar entornos volátiles. Cuadernos de Administración 26(47), 63-85.

Mendoza, J. M. (2013b). La estrategia y las capacidades dinámicas.Ide@s CONCYTEG, 8(99), 887-902.

Nishida, K. (1921). An Inquiry into the Good. M. Abe and C. Ives (trans.) (1990). New Haven/London: Yale University.

Nonaka, I., \& Takeuchi, H. (1995). The knowledge-creating company. Oxford: Oxford University Press.

Nonaka, I. \& Konno, N. (1998). The concept of "Ba": building a foundation for knowledge creation. California Management Review, 40(3), 40-54.

Nonaka, I., Toyama, R. \& Konno, N. (2000). SECl, Ba and Leadership: a Unified Model of Dynamic Knowledge Creation. Long Range Planning, 33, 5-34. 
Padua, J. (1979). Técnicas de Investigación aplicadas a las Ciencias Sociales. México D.F: Trillas.

Penrose, E. (1959). The theory of the growth of the firm. New York: John Wiley.

Robbins, S. y Judge, T. (2009). Comportamiento Organizacional (13a. ed.). (J. L. Brito, Trad.). México: Pearson-Prentice Hall.

Shapiro, S. (18 de 10 de 2005). Innovar para ser Competitivo. Obtenido de http://winred.com/EP/entrevistas/n/a2880.html

Shimizu, H. (1995). Ba-Principle: New Logic for the Real-time Emergence of Information, Holonics, 5(1), 67-69.

Schumpeter, J. A. (1934). The Theory of Economic Development. Cambridge, MA: Harvard University Press.

Shumpeter, J. (1978). Teoría de desenvolvimiento económico. (Quinta Reimpresión ed.). México: Fondo de Cultura Económica.

Subramaniam, M., \& Youndt, M. A. (2005). The influence of intellectual capital on the types of innovative capabilities. Academy of Management Journal, 48, 450-463.

Szeto, E. (2000). Innovation capacity: Working towards a mechanism for imimproving innovation within an inter-organizational network. The TQM Magazine, 12(2), 149-157.

Teece, D. (2007). Explicating dynamic capabilities: The nature and microfundations of (sustainable) enterprise performarmance. Strategic Management Journal, 28, 1319-1350.

Teece, D. (2009). Dynamic Capabilities \& Strategic Management. Organizing for Innovation an Growth. New York: Oxford University Press Inc.

Teece, D., Pisano, G., \& Shuen, A. (1997). Dynamic caapbilities and strategic management. Strategic Management Journal 18(7), 509-533.

Toffler, A. (1985). La empresa flexible. Obtenido de http://Es.Scribd.Com/ Doc/186730547/Alvin-Toffler-La-Empresa-Flexible-1985

Vivas, S. (2013). Implicaciones de las capacidades dinámicas para la com- 
petitividad y la innovación en el siglo XXI. Cuadernos de Administración, 26(47), 119-139.

Wang, C., \& Ahmed, P. (2007). Dynamic Capabilities: A review and Research Agenda. Strategic Management Journal, 9(1), 31-51.

Wernerfelt, B. (1984). A resource-based view of the firm. Strategic Management Journal, 5(2), 171-180.

Venture, V. (1994). Análisis competitivo de la empresa: un enfoque estratégico. Madrid: Civitas

Zhao, H. T. (2005). Types of technology sourcing and innovative capability: An exploratory study of Singapore manufacturmanufacturing. Journal of High Technology Management Research, 16, 209-224.

Zott, C. (2003). Dynamic capabilities and the emergence of intraindustry differential firm performance: Insights from a simulation study. Strategic Management Journal, 24(2), 97-125.

Winter, S. (2003). Understanding dynamic capabilties. Strategic Management Review, (24), 991-995.

Wolfe, R. (1994). Organizational innovation: Review, critique and suggested research directions. Journal of Management Studies, 31(3), 405-431.

Zahra, S. \& George, G. (2002). Absorptive capacity: A review, reconceptualisation, and extension. Academy of Management Review, 27(2), 185-203.

Cómo citar este capítulo:

Aquino Vanegas, B., Barrios Hernández, K., Olivero Vega, E., Mendoza Guerra, J. M. (2017). Gestión del conocimiento y capacidad de innovación en una organización radial en barranquilla. En K. Barrios Hernández, E. Olivero Vega, J. C. Acosta Prado, I. Meñaca Guerrero, A. M. Cazallo Antúnez, H. J. Medina Carrascal, ... O. Hernández Ariza, Gestión del conocimiento y capacidad de innovación. Modelos, Sistemas y Aplicaciones (pp.199-226). Barranquilla: Universidad Simón Bolívar. 